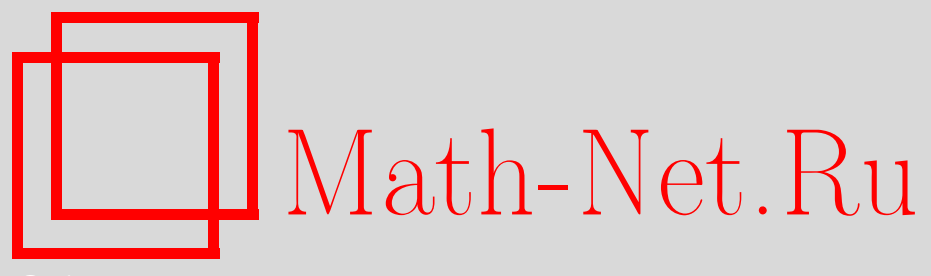

А. Д. Суханов, Соотношение неопределенностей Шредингера для квантового осциллятора в термостате, TMФ, 2006, том 148, номер 2, 295-308

DOI: https://doi.org/10.4213/tmf2087

Использование Общероссийского математического портала Math-Net.Ru подразумевает, что вы прочитали и согласны с пользовательским соглашением http: //www. mathnet.ru/rus/agreement

Параметры загрузки:

IP : 44.207 .124 .84

26 апреля 2023 г., 18:19:47

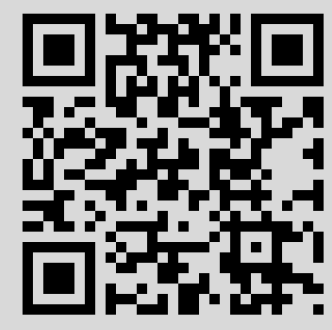




\title{
СООТНОШЕНИЕ НЕОПРЕДЕЛЕННОСТЕЙ ШРЕДИНГЕРА ДЛЯ КВАНТОВОГО ОСЦИЛЛЯТОРА В ТЕРМОСТАТЕ ${ }^{1)}$
}

\begin{abstract}
Введен коррелятор Шредингера как целостная характеристика двух типов корреляции флуктуаций в квантовой динамике и в статистической термодинамике. Впервые проведено его вычисление методами термополевой динамики для переменных координата-импульс квантового осциллятора в термостате. Показано, что полученное значение обеспечивает обращение соотношения неопределенностей Шредингера в равенство при любых температурах. Установлено, что состояние теплового равновесия для квантового осциллятора имеет смысл теплового коррелированно-когерентного состояния и может быть адекватно описано волновой функцией с амплитудой и фазой, зависящими от температуры.
\end{abstract}

Ключевые слова: коррелятор Шредингера, насыщенное соотношение неопределенностей Шредингера, неаддитивность квантовых и тепловых флуктуаций, тепловое коррелированно-когерентное состояние, тепловой шум в чистом состоянии.

\section{1. ПОСТАНОВКА ПРОБЛЕМЫ}

В открытых квантовых системах в состоянии теплового равновесия, в том числе в системах с небольшим числом степеней свободы, одновременно могут иметь место квантовые и тепловые флуктуации сопряженных физических величин [1]. До сих пор основное внимание уделялось анализу корреляций либо между флуктуациями микропараметров в квантовой динамике (при $T=0$ ) [2], [3], либо между флуктуациями макропараметров в статистической термодинамике [4], [5]. Было показано, что в обеих теориях существуют состояния, в которых корреляция сопряженных величин оказывается максимальной, что приводит к насыщению соответствующих соотношений неопределенностей. Однако в общем случае интересен анализ корреляций между флуктуациями сопряженных величин при одновременном проявлении

1) В статье использованы материалы доклада автора на Боголюбовской конференции "Проблемы теоретической и математической физики" (Дубна, ОИЯИ, 2-6 сентября 2004 г.)

*Российский университет дружбы народов, Москва, Россия. E-mail: ogol@oldi.ru 
квантовых и тепловых эффектов. По этому поводу в 1998 г. была выдвинута эвристическая гипотеза [6] о неаддитивном характере корреляции квантовых и тепловых флуктуаций.

Для проверки этой гипотезы выберем простейшую, но фундаментальную модель открытой квантовой системы - одномерный квантовый осциллятор в термостате. Распределения вероятности для такой модели в координатном и импульсном представлениях были получены Блохом еще в 1932 г. [7] (см. также [1], [8]). Из его результатов следует, что среднеквадратичные отклонения (вариации) импульса $p$ и координаты $q$ имеют вид

$$
\begin{aligned}
& \overline{(\Delta p)^{2}}=\overline{(\Delta p)_{0}^{2}} \operatorname{cth} \frac{\hbar \omega}{2 k_{\mathrm{B}} T}, \\
& \overline{(\Delta q)^{2}}=\overline{(\Delta q)_{0}^{2}} \operatorname{cth} \frac{\hbar \omega}{2 k_{\mathrm{B}} T},
\end{aligned}
$$

где

причем

$$
\overline{(\Delta p)_{0}^{2}}=\frac{\hbar \omega m}{2}, \quad \overline{(\Delta q)_{0}^{2}}=\frac{\hbar}{2 \omega m},
$$

$$
1 \leqslant \operatorname{cth} \frac{\hbar \omega}{2 k_{\mathrm{B}} T} \leqslant \frac{k_{\mathrm{B}} T}{\hbar \omega / 2} .
$$

Даже поверхностный взгляд на формулы (1), содержащие одинаковую функциональную зависимость от аргумента $\omega / T$, наводит на мысль о возможной зависимости импульса и координаты осциллятора в состоянии теплового равновесия. Поскольку, однако, это подозрение напрямую подтвердить не удается, можно пойти косвенным путем и наряду с величинами $\overline{(\Delta p)^{2}}$ и $\overline{(\Delta q)^{2}}$ вычислить еще одну характеристику распределения вероятности - коррелятор (ковариацию) импульса и координаты. Совместное рассмотрение всех трех моментов второго порядка позволит сделать вывод о зависимости величин $p$ и $q$ или об ее отсутствии.

Рассмотрим, например, неквантовый осциллятор в теории броуновского движения в состоянии полного теплового равновесия, достигаемом в пределе $t \gg \tau$, где $\tau$ - время релаксации. Для этого осциллятора коррелятор (ковариация) $\sigma_{p q}$ импульса и координаты, вычисленный с помощью методов теории вероятности, имеет вид [9]

$$
\sigma_{p q} \equiv \overline{(\Delta p \Delta q)}=\frac{k_{\mathrm{B}} T}{\omega} .
$$

Здесь усреднение производится по распределению $\rho(q)$, имеющему форму распределения Гаусса с $\overline{(\Delta q)^{2}}=k_{\mathrm{B}} T /\left(m \omega^{2}\right)$ и являющемуся решением уравнения диффузии. Фурье-образ этого распределения также имеет вид распределения Гаусса $\rho(p)$ с $\overline{(\Delta p)^{2}}=m k_{\mathrm{B}} T$. Отличие от нуля коррелятора $\sigma_{p q}$ в (2) служит неким указанием на зависимость между импульсом и координатой броуновского осциллятора, обусловленную тепловым воздействием со стороны термостата.

В качестве другого примера рассмотрим квантовый осциллятор при $T=0$. Как известно, операторы $\hat{p}$ и $\hat{q}$ не коммутируют. Это обстоятельство также можно трактовать как своеобразную корреляцию импульса и координаты. Ее характеристикой служит величина

$$
c_{p q}=\frac{1}{2}|\langle|[\hat{p}, \hat{q}]|\rangle|=\frac{\hbar}{2},
$$


входящая в правую часть соотношения неопределенностей Гейзенберга [3], в котором она выступает на равных со среднеквадратичными отклонениями импульса $\overline{(\Delta p)^{2}}$ и координаты $\overline{(\Delta q)^{2}}$. Отличие от нуля величины $c_{p q}$ в $(3)$, как и выше, служит неким указанием на зависимость между импульсом и координатой квантового осциллятора, обусловленную в данном случае квантовым воздействием макроокружения. Таким образом, величины $\sigma_{p q}$ и $c_{p q}$ в этих примерах можно рассматривать как характеристики зависимости между импульсом и координатой осциллятора при наличии чисто тепловых или чисто квантовых эффектов.

Цель данной работы состоит в решении следующих задач:

- получить общее выражение для характеристики взаимозависимости импульса и координаты осциллятора при одновременном наличии квантовых и тепловых эффектов;

- убедиться в том, что соотношение неопределенностей Шредингера для квантового осциллятора в термостате является насыщенным при любой температуре;

- показать, что состояние теплового равновесия может быть интерпретировано как тепловое коррелированно-когерентное состояние, у которого амплитуда и фаза волновой функции зависят от температуры.

\section{2. КОРРЕЛЯТОР ШРЕДИНГЕРА}

Наиболее общая характеристика $\widetilde{R}_{p q}$ корреляции пары сопряженных величин $p$ и $q$ в квантовой динамике была введена Шредингером [10], [11] как амплитуда перехода из состояния $|\Delta q\rangle$ в состояние $|\Delta p\rangle$ :

$$
\widetilde{R}_{p q}=\langle\Delta p \mid \Delta q\rangle=\langle|\Delta \hat{p} \Delta \hat{q}|\rangle,
$$

где

$$
\begin{array}{ll}
|\Delta p\rangle=\Delta \hat{p}|\rangle, & \Delta \hat{p}=\hat{p}-\langle|\hat{p}|\rangle, \\
|\Delta q\rangle=\Delta \hat{q}|\rangle, & \Delta \hat{q}=\hat{q}-\langle|\hat{q}|\rangle .
\end{array}
$$

Комплексную величину $\widetilde{R}_{p q}$ мы будем впредь называть коррелятором Шрединге$p a$. Следуя Шредингеру, ее можно представить в виде

$$
\widetilde{R}_{p q}=\frac{1}{2}\langle|\Delta \hat{p} \Delta \hat{q}-\Delta \hat{q} \Delta \hat{p}|\rangle+\frac{1}{2}\langle|\Delta \hat{p} \Delta \hat{q}+\Delta \hat{q} \Delta \hat{p}|\rangle .
$$

Первое слагаемое в формуле (6) в соответствии с формулой (3) можно представить как $-i c_{p q}$ (величины $\bar{q}=\langle|\hat{q}|\rangle$ и $\bar{p}=\langle|\hat{p}|\rangle$ из него выпадают). Нетрудно видеть, что второе слагаемое в квазиклассическом пределе имеет смысл ковариации $(\overline{\Delta p \Delta q})$, и потому его можно здесь также обозначить $\sigma_{p q}$. Следовательно, коррелятор Шредингера можно представить в виде

$$
\widetilde{R}_{p q}=-i\left(c_{p q}+i \sigma_{p q}\right) .
$$

Величина $c_{p q}$ в выражении (7) отражает только один из типов корреляции импульса и координаты, связанный, как уже отмечалось, с некоммутативностью соответствующих операторов. Можно предположить, что в общем случае величина 
$\sigma_{p q}$ соответствует другому типу корреляции, в чем-то аналогичному корреляции в формуле (2) в теории броуновского движения.

Действительно, величина $\sigma_{p q}$ в координатном представлении имеет вид [3]

$$
\sigma_{p q}=m \int d q(q-\bar{q}) j_{\operatorname{prob}}(q)
$$

где $j_{\text {prob }}(q)$ - плотность потока вероятности. В квазиклассическом пределе $\sigma_{p q}$ переходит в ковариацию $(\overline{\Delta p \Delta q})$ из теории вероятностей. Поскольку в этом пределе величина $j_{\operatorname{prob}}(q)$ переходит в плотность потока частиц $j_{n}(q)$, для броуновского движения ковариация выражается (см. [5]) через коэффициент диффузии $\left(\sigma_{p q}=m D\right)$, что для броуновского осциллятора приводит к формуле (2) [9].

Таким образом, коррелятор Шредингера в общем случае имеет смысл характеристики двух типов корреляции сопряженных величин $p$ и $q$, один из которых связан с коммутатором, а другой - с антикоммутатором (или ковариацией).

Сопоставление двух типов корреляции импульса и координаты удобно провести, использовав стохастическую механику, предложенную Нельсоном [12], как версию квантовой механики. Записав волновую функцию в виде

$$
\psi(q)=\sqrt{\varrho_{\text {prob }}(q)} e^{i \varphi(q)},
$$

для коррелятора Шредингера $\widetilde{R}_{p q}$ получим выражение [3]

$$
\widetilde{R}_{p q}=-i\left(c_{p q}+i \sigma_{p q}\right)=-i[m(\overline{q U})+i m(\overline{q V})] .
$$

В нем каждое из слагаемых $c_{p q}$ и $\sigma_{p q}$ выражается через одну из характерных скоростей $V(q)$ и $U(q)$, введенных Нельсоном, и вычисляется как ковариация в рамках классической теории вероятности, причем распределение имеет вид $\rho_{\text {prob }}(q)=$ $|\psi(q)|^{2}$. При этом скорость $U(q)$ определяется вещественной амплитудой волновой функции (9),

$$
U(q)=-\frac{\hbar}{m} \frac{\partial}{\partial q} \ln \sqrt{\rho_{\text {prob }}(q)}
$$

а скорость $V(q)$ - фазой той же волновой функции,

$$
V(q) \equiv \frac{\hbar}{m} \frac{\partial \varphi}{\partial q}
$$

Очевидно, что в случае вещественных волновых функций, когда фаза $\varphi=0$, связанное с ней слагаемое в корреляторе Шредингера $\sigma_{p q}=0$. При этом вся корреляция импульса и координаты сводится к слагаемому $c_{p q}$, отражающему в этом случае некоммутативность операторов $\hat{p}$ и $\hat{q}$ лишь косвенно. Соответственно в квазиклассическом пределе, наоборот, слагаемое $c_{p q} \rightarrow 0$, так что корреляция тех же величин определяется только слагаемым $\sigma_{p q} \neq 0$. В общем случае коррелятор Шредингера целостно характеризует взаимозависимость импульса и координаты. Интересно, что в терминах стохастической механики оба типа корреляции дают единообразные вклады в $\widetilde{R}_{p q}$, что подчеркивает их равноправную роль в корреляторе Шредингера в целом. 
Среди множества состояний микросистемы физически выделены состояния, в которых корреляция величин $p$ и $q$ оказывается наиболее высокой. В них модуль коррелятора Шредингера $\left|\widehat{R}_{p q}\right|$ оказывается максимальным. Эти состояния принято называть коррелированно-когерентными состояниями [13]. Волновая функция произвольной микросистемы, соответствующая коррелированно-когерентному состоянию, имеет вид комплексного распределения Гаусса [3], [13]:

$$
\psi(q)=\left[2 \pi \overline{(\Delta q)^{2}}\right]^{-1 / 4} \exp \left\{-\frac{q^{2}}{4 \overline{(\Delta q)^{2}}}(1-i \alpha)\right\},
$$

где $\alpha$ - параметр, фиксирующий тот или иной выбор класса подобных состояний для данной микросистемы, а $\overline{\Delta q^{2}}$ - среднеквадратичное отклонение координаты (вариация), вычисленное по распределению $\rho_{\text {prob }}(q)=|\psi(q)|^{2}$. Как показано в [3], вычисление коррелятора Шредингера $\widetilde{R}_{p q}$ в рамках либо обычной квантовой механики с учетом (13), либо стохастической механики Нельсона с учетом (10)-(12) приводит к одному и тому же результату:

$$
\widetilde{R}_{p q}=-i\left(c_{p q}+i \sigma_{p q}\right)=-i\left(\frac{\hbar}{2}+i \frac{\hbar}{2} \alpha\right) .
$$

Проверим, что в состоянии, характеризуемом волновой функцией вида (13) с произвольным параметром $\alpha$, коррелятор Шредингера действительно является максимальным. Иными словами, убедимся, что в этом случае соотношение неопределенностей Шредингера достигает насыщения, т.е. становится равенством.

Стоящее в левой части соотношения неопределенностей произведение среднеквадратичных флуктуаций импульса и координаты, имеющее размерность квадрата действия, представляет собой целостную величину, которую в работе [14] предложено называть the uncertainties product (UP),

$$
\mathcal{U P} \equiv \overline{(\Delta p)^{2}} \cdot \overline{(\Delta q)^{2}} .
$$

В этих терминах соотношение неопределенностей Шредингера принимает вид

$$
\mathcal{U P} \geqslant\left|\widetilde{R}_{p q}\right|^{2} .
$$

В данном случае величина $\overline{(\Delta p)^{2}}$, вычисленная с помощью $\rho_{\text {prob }}(q)$, такова:

$$
\overline{(\Delta p)^{2}}=\frac{\hbar^{2}}{4} \frac{\alpha^{2}+1}{\overline{(\Delta q)^{2}}} .
$$

Поэтому выражения для $\mathcal{U P}$ и коррелятора $\widetilde{R}_{p q}$, вычисленные независимо на основании формул (15), (17) и формулы (14), соответственно, принимают вид

$$
\begin{aligned}
\mathcal{U P} & \equiv \frac{\hbar^{2}}{4}\left(\alpha^{2}+1\right), \\
\left|\widetilde{R}_{p q}\right|^{2} & \equiv c_{p q}^{2}+\sigma_{p q}^{2}=\frac{\hbar^{2}}{4}+\frac{\hbar^{2}}{4} \alpha^{2} .
\end{aligned}
$$


Отсюда следует, что, как и ожидалось, соотношение неопределенностей Шредингера является насыщенным при любом выборе параметра $\alpha$,

$$
\mathcal{u P}=\left|\widetilde{R}_{p q}\right|^{2} .
$$

При $\alpha=0$ коррелятор Шредингера (14) переходит в чисто мнимую величину $-i c_{p q}$. В этом случае соотношение неопределенностей (18), оставаясь равенством, сводится к соотношению неопределенностей Гейзенберга:

$$
\mathcal{u P} \equiv \frac{\hbar^{2}}{4}=c_{p q}^{2} \equiv \frac{\hbar^{2}}{4} .
$$

\section{3. КОРРЕЛЯТОР ШРЕДИНГЕРА ДЛЯ КВАНТОВОГО ОСЦИЛЛЯТОРА В КОРРЕЛИРОВАННО-КОГЕРЕНТНЫХ СОСТОЯНИЯХ}

Как известно, соотношение неопределенностей в виде равенства (19) характерно для основного состояния квантового осциллятора. Однако для других его когерентных состояний или для состояний с определенной энергией равенство (19) превращается в строгое неравенство, поскольку в нем величина UР увеличивается, а коррелятор Шредингера $\widetilde{R}_{p q}=-i c_{p q}$ остается неизменным [3], [11]. Это свидетельствует о том, что в таких состояниях, как и в основном состоянии, $\sigma_{p q}=0$, т.е. имеет место только простейшая корреляция квантового типа, связанная с некоммутативностью соответствующих операторов.

Оба типа квантовой корреляции для осциллятора могут иметь место в коррелированно-когерентных состояниях, которые удается получить из обычных когерентных состояний с помощью $(u, v)$-преобразования Боголюбова [13]. Оно позволяет конкретизировать выражения для величин $\alpha$ и $\overline{\Delta q^{2}}$, входящих в случае осциллятора в волновую функцию вида (13).

С этой целью перейдем от совокупности операторов рождения $\hat{a}^{\dagger}$ и уничтожения $\hat{a}$ к совокупности других операторов рождения $\hat{b}^{\dagger}$ и уничтожения $\hat{b}$ с соответствующим изменением вакуума:

$$
\hat{b}=u \hat{a}+v \hat{a}^{\dagger}, \quad \hat{b}^{\dagger}=v^{*} \hat{a}+u^{*} \hat{a}^{\dagger},
$$

где $|u|^{2}-|v|^{2}=1$. Заметим, что в рамках обычной квантовой механики $(u, v)$ преобразование Боголюбова приводит к унитарно эквивалентному представлению канонических перестановочных соотношений.

Традиционный выбор параметризации функций $u$ и $v$,

$$
u=e^{i \chi} \operatorname{ch} \tau, \quad v=e^{-i(\theta+\chi)} \operatorname{sh} \tau
$$

при $0 \leqslant \tau<\infty,-\pi \leqslant \chi \leqslant \pi, \theta=0$, позволяет в общем случае найти выражения для величин $\alpha$ и $\overline{(\Delta q)^{2}}$ в волновой функции (13). Как показано в [3], эти выражения таковы:

$$
\begin{gathered}
\alpha=\operatorname{sh} 2 \tau \sin 2 \chi, \\
\overline{(\Delta q)^{2}}=\frac{\hbar}{2 m \omega}\left(e^{-2 \tau}+2 \operatorname{sh} 2 \tau \cdot \sin ^{2} \chi\right),
\end{gathered}
$$


так что с учетом общих формул (17) и (14) получим

$$
\begin{gathered}
\overline{(\Delta p)^{2}}=\frac{\hbar \omega m}{2} \frac{\operatorname{sh}^{2} 2 \tau \sin ^{2} 2 \chi+1}{e^{-2 \tau}+2 \operatorname{sh} 2 \tau \sin ^{2} \chi} \\
\left|\widetilde{R}_{p q}\right|^{2}=\frac{\hbar^{2}}{4}+\frac{\hbar^{2}}{4} \operatorname{sh}^{2} 2 \tau \sin ^{2} 2 \chi
\end{gathered}
$$

Таким образом, для квантового осциллятора в термостате величины $\alpha$ и $\overline{(\Delta q)^{2}}$ в волновой функции $\psi(q)$ вида (13) могут быть выражены через параметры $\tau$ и $\chi$, характеризующие $(u, v)$-преобразование Боголюбова.

Подчеркнем, что выражение (25) по-прежнему соответствует корреляции квантового типа. Однако оно содержит два качественно различных вклада $c_{p q}$ и $\sigma_{p q}$ в коррелятор Шредингера $\widetilde{R}_{p q}$, обусловленные соответственно коммутатором и антикоммутатором. Вместе с тем в квазиклассическом пределе $(\alpha \gg 1)$ сохраняется только вклад $\sigma_{p q}$. Аналогичный вклад $(2)$ имеет место и в случае неквантового осциллятора в теории броуновского движения, отражая наличие теплового воздействия термостата. Приравнивая в этом пределе, т.е. при высоких температурах, величину $\sigma_{p q}=\hbar \alpha_{\text {quasi }} / 2$ из формулы (14) к величине $\sigma_{p q}$ из формулы $(2)$, получим, что

$$
\alpha_{\text {quasi }}=\frac{k_{\mathrm{B}} T}{\hbar \omega / 2} \gg 1
$$

Это позволяет предположить, что квазиклассическое приближение в описании квантового осциллятора в термостате соответствует описанию неквантового осциллятора в теории броуновского движения (при $t \gg \tau$ ). Дополнительным аргументом в пользу этого предположения служит совпадение выражения для $\mathcal{U P}$, вычисленного с помощью распределения Гаусса в теории броуновского движения, с выражением для $\mathcal{U P}$, вытекающим при $k_{\mathrm{B}} T \gg \hbar \omega / 2$ из формул (1) для квантового осциллятора в термостате: в случае неквантового броуновского осциллятора

$$
\mathcal{U P}=\left(m k_{\mathrm{B}} T\right) \cdot \frac{k_{\mathrm{B}} T}{m \omega^{2}}=\left(\frac{k_{\mathrm{B}} T}{\omega}\right)^{2} ;
$$

в случае квантового осциллятора в квазиклассическом пределе

$$
\mathcal{U P}=\frac{\hbar \omega m}{2} \operatorname{cth}\left(\frac{1}{\alpha_{\text {quasi }}}\right) \cdot \frac{\hbar}{2 m \omega} \operatorname{cth}\left(\frac{1}{\alpha_{\text {quasi }}}\right) \rightarrow\left(\frac{\hbar}{2} \alpha_{\text {quasi }}\right)^{2} .
$$

Таким образом, в пределе высоких температур с учетом формул (2) и (27) соотношение неопределенностей Шредингера для квантового осциллятора в термостате принимает вид

$$
\mathcal{u P} \equiv\left(\frac{k_{\mathrm{B}} T}{\omega}\right)^{2}=\sigma_{p q}^{2} \equiv\left(\frac{k_{\mathrm{B}} T}{\omega}\right)^{2}
$$

т.е. является насыщенным. Примем во внимание, что основное состояние квантового осциллятора (с $\alpha=0)$, для которого соотношение неопределенностей (19) также является насыщенным, соответствует равновесному состоянию при нулевой 
температуре термостата. Поэтому представляется естественным предположить, что данное свойство сохраняется и в промежуточной области температур. Однако стандартные расчеты с помощью матрицы плотности, т.е. с использованием смешанных состояний, этого не подтверждают [1].

\section{4. КОРРЕЛЯТОР ШРЕДИНГЕРА ДЛЯ КВАНТОВОГО ОСЦИЛЛЯТОРА В ТЕРМОПОЛЕВОЙ ДИНАМИКЕ}

Из проведенного выше анализа следует, что насыщение соотношения неопределенностей Шредингера имеет место в произвольных коррелированно-когерентных состояниях, описываемых волновой функцией (13) с параметрами (22) и (23). Если осциллятор помещен в термостат, то эти параметры, по крайней мере в квазиклассическом пределе, зависят от температуры. Стандартный аппарат, основанный на матрице плотности, оказывается здесь неэффективным. В связи с этим попробуем найти описание квантового осциллятора в термостате, основанное на использовании чистого состояния, но при этом будем считать, что амплитуда и фаза волновой функции явно зависят от температуры.

Отметим,что полученные выше величины $\overline{(\Delta p)^{2}}$ и $\overline{(\Delta q)^{2}}$ (в отличие от величин, заданных в (1)) имеют в общем случае вид совершенно различных функций (24) и (23) параметров $\tau$ и $\chi$. Разумеется, обе они, как впрочем, и $\widetilde{R}_{p q}$, не зависят от температуры, так что пока их нельзя сопоставить состоянию теплового равновесия. Чтобы этого добиться, нужно решить две проблемы: во-первых, выбрать параметры $\tau, \chi$ так, чтобы выражения (24) и $(23)$ для величин $\overline{(\Delta p)^{2}}$ и $\overline{(\Delta q)^{2}}$ приобрели одинаковую функциональную зависимость; во-вторых, найти возможность связать эти параметры с температурой, установив тем самым некое соответствие между описаниями осциллятора в термостате с использованием чистых и смешанных состояний.

Первая проблема решается относительно просто. Действительно, если выбрать $\chi=\pi / 4$, то искомые выражения для параметров $\alpha$ и $\overline{(\Delta q)^{2}}$ вместо (22) и (23) примут вид

$$
\begin{gathered}
\alpha=\operatorname{sh} 2 \tau, \\
\overline{(\Delta q)^{2}}=\frac{\hbar}{2 m \omega}\left(e^{-2 \tau}+\operatorname{sh} 2 \tau\right)=\frac{\hbar}{2 m \omega} \operatorname{ch} 2 \tau .
\end{gathered}
$$

Тогда из формул (24) и (25) получим

$$
\begin{gathered}
\overline{(\Delta p)^{2}}=\frac{\hbar^{2}}{4} \frac{2 m \omega}{\hbar} \frac{\operatorname{sh}^{2} 2 \tau+1}{\operatorname{ch} 2 \tau}=\frac{\hbar \omega m}{2} \operatorname{ch} 2 \tau, \\
\left|\widetilde{R}_{p q}\right|^{2}=\frac{\hbar^{2}}{4}+\frac{\hbar^{2}}{4} \operatorname{sh}^{2} 2 \tau=\frac{\hbar^{2}}{4} \operatorname{ch}^{2} 2 \tau .
\end{gathered}
$$

Чтобы решить вторую проблему, необходимо связать функцию $\operatorname{ch} 2 \tau$, входящую, в частности, в формулы (31) и $(30)$, с функцией $\operatorname{cth}\left(\hbar \omega / 2 k_{\mathrm{B}} T\right)$, входящей в соответствующие им формулы в (1). Однако в рамках исходного гильбертового пространства состояний этого сделать нельзя, ибо вследствие унитарной эквивалентности соответствующих представлений операторов рождения и уничтожения чистые состояния квантового осциллятора не могут сами собой превратиться в смешанные. 
Выход из создавшегося положения предоставляет термополевая динамика Умэдзавы [15], [16] как одна из версий квантовой теории поля при конечных температурах.

Напомним, что главная физическая идея термополевой динамики состоит в следующем: в результате помещения системы в термостат вместо одного механизма поглощения системой энергии извне эффективно проявляются два независимых механизма - за счет возбуждения новых квантов и за счет исчезновения вакансий, появившихся в спектре системы под влиянием термостата. Иными словами, происходит удвоение числа типов степеней свободы. Чтобы их описать на языке чистых состояний, предлагается ввести удвоенное гильбертово пространство $\mathfrak{H} \otimes \mathfrak{H}$. В представлении чисел заполнения оно определяется двойным набором операторов рождения и уничтожения, включающим как исходные операторы $\hat{a}$ и $\hat{a}^{\dagger}$, отвечающие динамическим степеням свободы, так и операторы $\hat{\tilde{a}}$ и $\hat{\tilde{a}}^{\dagger}$, отвечающие тепловым степеням свободы.

Для получения коррелированно-когерентных состояний в таком пространстве используется более общее $(u, v)$-преобразование Боголюбова, чем преобразование (20) [16]. В результате его применения от набора операторов $\hat{a}, \hat{a}^{\dagger}$ и $\hat{\tilde{a}}, \hat{\tilde{a}}^{\dagger}$ для исходных частиц переходят к другому набору операторов $\hat{b}, \hat{b}^{\dagger}$ и $\hat{\tilde{b}}, \hat{\tilde{b}}^{\dagger}$ для квазичастиц по формулам

$$
\hat{b}=u \hat{a}+v \hat{\tilde{a}}^{\dagger}, \quad \tilde{b}=u \hat{\tilde{a}}+v \hat{a}^{\dagger}
$$

и по аналогичным формулам для операторов $\hat{b}^{\dagger}$ и $\hat{\tilde{b}}^{\dagger}$ с теми же, что и выше, функциями $u$ и $v$. Два качественно различных вакуума $|0\rangle\rangle$ и $|0(\tau)\rangle$ определяются условиями

$$
\hat{a}|0\rangle\rangle=\hat{\tilde{a}}|0\rangle\rangle=0, \quad \hat{b}(\tau)|0(\tau)\rangle=\hat{\tilde{b}}|0(\tau)\rangle=0 .
$$

Вакуум $|0(\tau)\rangle$ по отношению к исходным квантам $(a, \tilde{a})$ является конденсатом. Будучи ортогональным всем векторам исходного фокова пространства, данный вектор сам не принадлежит этому пространству. Иными словами, хотя преобразование (33) является каноническим, в данном случае оно приводит к унитарно неэквивалентному представлению канонических перестановочных соотношений, учитывающему тот факт, что термостат - это система с бесконечно большим числом степеней свободы.

Основная аксиома термополевой динамики [16], позволяющая сопоставить описания на языке чистых и смешанных состояний, исходит из идеи метода квазисредних Боголюбова. Предполагается, что воздействие термостата можно учесть двумя эквивалентными способами. Можно учесть всю зависимость от температуры, введя согласно преобразованию (33) зависящие от температуры через параметр $\tau$ операторы $\hat{b}(\tau), \hat{b}^{\dagger}(\tau)$ и $\hat{\tilde{b}}(\tau), \hat{\tilde{b}}^{\dagger}(\tau)$ в удвоенном гильбертовом пространстве, но производя усреднение по чистому состоянию - вакууму $|0\rangle\rangle$, не зависящему от температуры. Ту же зависимость от температуры можно учесть и другим способом, используя исходные (не зависящие от температуры) операторы $\hat{a}, \hat{a}^{\dagger}$ и $\hat{\tilde{a}}, \hat{\tilde{a}}^{\dagger}$, но при этом производя усреднение по смешанному состоянию, описываемому матрицей плотности, 
зависящей от температуры,

$$
\hat{\rho}(T)=[\operatorname{Tr} \hat{\rho}(T)]^{-1} \exp \left\{-\frac{\widehat{H}}{k_{\mathrm{B}} T}\right\} ; \quad \widehat{H}=\hbar \omega\left(\hat{a}^{\dagger} \hat{a}+\frac{1}{2}\right) .
$$

При этом требуется, чтобы среднее от оператора числа квазичастиц $\widehat{N}(\tau)=$ $\hat{b}^{\dagger}(\tau) \hat{b}(\tau)$ по исходному вакууму было равно среднему от числа исходных частиц $\widehat{N}=$ $\hat{a}^{\dagger} \hat{a}$ по распределению Гиббса с оператором плотности $\hat{\rho}(T)$ вида (35). Иными словами, оставшийся произвольным параметр $\tau$, характеризующий $(u, v)$-преобразование Боголюбова (33), предлагается фиксировать равенством

$$
\left\langle\left\langle 0\left|\hat{b}^{\dagger}(\tau) \hat{b}(\tau)\right| 0\right\rangle\right\rangle=\operatorname{Tr}\left[\left(\hat{a}^{\dagger} \hat{a}\right) \hat{\rho}(T)\right] .
$$

Подставляя в левую часть этого равенства выражение (33) для $\hat{b}$ и соответствующее выражение для $\hat{b}^{\dagger}$ с неизвестной зависимостью $\tau=f(T)$, получим

$$
\left\langle\left\langle 0\left|\left(u^{*} \hat{a}^{\dagger}+v^{*} \hat{\tilde{a}}\right)\left(u \hat{a}+v \hat{\tilde{a}}^{\dagger}\right)\right| 0\right\rangle\right\rangle=|v|^{2}\left\langle\left\langle 0\left|\hat{\tilde{a}} \hat{\tilde{a}}^{\dagger}\right| 0\right\rangle\right\rangle=|v|^{2} .
$$

В свою очередь, правую часть равенства (36) проще всего можно получить, выразив ее через среднее значение энергии осциллятора в термостате

$$
\begin{aligned}
\bar{\varepsilon} & =\operatorname{Tr}(\widehat{H} \hat{\rho}(T))=\operatorname{Tr}\left\{\left[\hbar \omega\left(\widehat{N}+\frac{1}{2}\right)\right] \hat{\rho}(T)\right\}= \\
& =\frac{\hbar \omega}{2}+\frac{\hbar \omega}{e^{\hbar \omega / k_{\mathrm{B}} T}-1}=\frac{\hbar \omega}{2} \operatorname{cth} \frac{\hbar \omega}{2 k_{\mathrm{B}} T} .
\end{aligned}
$$

Отсюда следует, что

$$
\operatorname{Tr}(\widehat{N} \hat{\rho}(T))=\frac{\bar{\varepsilon}-\hbar \omega / 2}{\hbar \omega}=\frac{1}{2} \operatorname{cth} \frac{\hbar \omega}{2 k_{\mathrm{B}} T}-\frac{1}{2} .
$$

Подставляя выражения (37) и (39) в равенство (36), получаем искомую взаимосвязь параметра преобразования Боголюбова $\tau$ с температурой термостата $T$ :

$$
|v|^{2}=\operatorname{sh}^{2} \tau=\frac{1}{2} \operatorname{cth} \frac{\hbar \omega}{2 k_{\mathrm{B}} T}-\frac{1}{2} .
$$

Следовательно,

$$
\operatorname{ch}^{2} \tau=1+\operatorname{sh}^{2} \tau=\frac{1}{2} \operatorname{cth} \frac{\hbar \omega}{2 k_{\mathrm{B}} T}+\frac{1}{2} .
$$

В результате для интересующих нас функций получаем выражения

$$
\begin{aligned}
\operatorname{ch} 2 \tau & =2 \operatorname{ch}^{2} \tau-1=\operatorname{cth} \frac{\hbar \omega}{2 k_{\mathrm{B}} T} \\
\operatorname{sh} 2 \tau=2 \operatorname{sh} \tau \operatorname{ch} \tau & =2\left[\left(\frac{1}{2} \operatorname{cth} \frac{\hbar \omega}{2 k_{\mathrm{B}} T}-\frac{1}{2}\right)\left(\frac{1}{2} \operatorname{cth} \frac{\hbar \omega}{2 k_{\mathrm{B}} T}+\frac{1}{2}\right)\right]^{1 / 2}= \\
& =\left(\operatorname{cth}^{2} \frac{\hbar \omega}{2 k_{\mathrm{B}} T}-1\right)^{1 / 2}=\frac{1}{\operatorname{sh}\left(\hbar \omega / 2 k_{\mathrm{B}} T\right)}
\end{aligned}
$$


Итак, поставленная цель достигнута. С учетом полученной связи (40) между параметрами $\tau$ и $T$, формулы $(31)$ и (30) для величин $\overline{(\Delta p)^{2}}$ и $\overline{(\Delta q)^{2}}$ совпадают с соответствующими формулами в $(1)$. Для коррелятора Шредингера $\widetilde{R}_{p q}$ имеет место выражение

$$
\widetilde{R}_{p q}=-i\left[\frac{\hbar}{2}+i \frac{\hbar}{2} \frac{1}{\operatorname{sh}\left(\hbar \omega / 2 k_{\mathrm{B}} T\right)}\right],
$$

откуда следует, что

$$
\left|\widetilde{R}_{p q}\right|^{2}=\frac{\hbar^{2}}{4}+\frac{\hbar^{2}}{4} \cdot \frac{1}{\operatorname{sh}^{2}\left(\hbar \omega / 2 k_{\mathrm{B}} T\right)}=\frac{\hbar^{2}}{4} \operatorname{cth}^{2} \frac{\hbar \omega}{2 k_{\mathrm{B}} T} .
$$

Очевидно, что величина $\left|\widetilde{R}_{p q}\right|^{2}$, заданная выражением (45), совпадает с UР и тем самым обеспечивает равенство в соотношении неопределенностей Шредингера при любых температурах:

$$
\mathcal{U P}=\left|\widetilde{R}_{p q}\right|^{2} .
$$

Таким образом, предложенный выше метод вычислений впервые позволил продемонстрировать насыщение этого соотношения для квантового осциллятора в термостате, что предсказывалось ранее рядом исследователей на основании интуитивных соображений (см., например, [17]). При этом продемонстрировано, что состояние теплового равновесия в термостате имеет смысл теплового коррелированнокогерентного состояния [16].

В пределе низких температур $\left(\hbar \omega / 2 \gg k_{\mathrm{B}} T\right)$ коррелятор Шредингера (44) переходит в $-i \hbar / 2$, а в пределе высоких температур $\left(\hbar \omega / 2 \ll k_{\mathrm{B}} T\right)$ - в ковариацию $\sigma_{p q}=k_{\mathrm{B}} T / \omega$, совпадающую с соответствующим выражением (2) из теории броуновского движения.

При произвольной температуре

$$
\left|\widetilde{R}_{p q}\right|=\left|\widetilde{R}_{p q}\right|_{T=0} \operatorname{cth} \frac{\hbar \omega}{2 k_{\mathrm{B}} T} \geqslant\left|\widetilde{R}_{p q}\right|_{T=0}=\frac{\hbar}{2},
$$

так что помещение квантового осциллятора в термостат соответствует эффективному увеличению модуля коррелятора Шредингера, имеющего размерность действия.

Иными словами, при наличии теплового воздействия корреляция величин $p$ и $q$ усиливается. Однако из двух слагаемых $c_{p q}$ и $\sigma_{p q}$, входящих в общем случае в величину $\widetilde{R}_{p q}$, это воздействие сказывается только на величине $\sigma_{p q}$, которая зависит и от $\hbar$, и от $k_{\mathrm{B}}$. При этом выражение $(47)$ для $\left|\widetilde{R}_{p q}\right|$, как исходно и предполагалось в работе [6], отнюдь не является аддитивным по отношению к воздействиям квантового и теплового типов.

Заметим, что относительная роль теплового и квантового воздействий сказывается не только на модуле комплексной величины $\widetilde{R}_{p q}$, но и на ее фазе $\gamma$. Действительно,

$$
\widetilde{R}_{p q}=\left|\widetilde{R}_{p q}\right| e^{i \gamma}
$$

где

$$
\gamma=\operatorname{arctg} \frac{\sigma_{p q}}{c_{p q}}=\operatorname{arctg}\left[\frac{1}{\operatorname{sh}\left(\hbar \omega / 2 k_{\mathrm{B}} T\right)}\right] .
$$

6 Теоретическая и математическая физика, т. 148, № 2, 2006 г. 
Фаза $\gamma$ меняется от нуля при низких температурах, когда воздействие является чисто квантовым, до $\pi / 2$ при высоких температурах, когда воздействие можно считать чисто тепловым.

\section{5. ЗАВИСИМОСТЬ ВОЛНОВОЙ ФУНКЦИИ ОСЦИЛЛЯТОРА В ТЕРМОСТАТЕ ОТ ТЕМПЕРАТУРЫ}

Если вернуться к волновой функции коррелированно-когерентного состояния вида (13), то для квантового осциллятора в термостате ее вещественная амплитуда $|\psi(q)|$ и ее фаза $\varphi(q)$ в соответствии с формулами (29), (30) и (43), (42) зависят от температуры. Что касается вещественной амплитуды, то она приводит к распределению вероятностей в координатном представлении $\rho_{\operatorname{prob}}(q)=|\psi(q)|^{2}$, впервые полученному с помощью матрицы плотности Ф. Блохом [7] (см. также [1], [8]):

$$
\rho(q)=\left[\frac{\pi \hbar}{m \omega} \operatorname{cth} \frac{\hbar \omega}{2 k_{\mathrm{B}} T}\right]^{-1 / 2} \exp \left\{-\frac{m \omega q^{2}}{\hbar \operatorname{cth}\left(\hbar \omega / 2 k_{\mathrm{B}} T\right)}\right\} .
$$

Для фазы $\varphi(q)$ имеем

$$
\varphi(q)=\frac{q^{2}}{4 \overline{(\Delta q)^{2}}} \alpha=\frac{m \omega q^{2}}{2 \hbar} \frac{1}{\operatorname{ch}\left(\hbar \omega / 2 k_{\mathrm{B}} T\right)} .
$$

Нетрудно видеть, что при $T \rightarrow 0$ фаза $\varphi \rightarrow 0$, так что волновая функция основного состояния, как и следовало ожидать, оказывается вещественной. В то же время при высоких температурах $k_{\mathrm{B}} T \gg \hbar \omega / 2$, когда квантовое воздействие, казалось бы, не должно сказываться,

$$
\varphi(q) \rightarrow \frac{m \omega q^{2}}{\hbar} \neq 0
$$

Таким образом, хотя это несколько неожиданно, роль фазы волновой функции сохраняется и в области, традиционно считавшейся классической. Именно благодаря этому обстоятельству удается получить предельное выражение для ковариации $\sigma_{p q}$, соответствующее аналогичному выражению (2) из теории броуновского движения (или теории тепловой диффузии). Действительно, согласно формулам (13) и (12), используя выражение (52), получим

$$
\sigma_{p q}=m \overline{(q V)}=m \frac{\hbar}{m} \overline{q \frac{\partial \varphi}{\partial q}}=\hbar \frac{1}{2 \hbar} 2 \overline{(m \omega q) q}=\frac{k_{\mathrm{B}} T}{\omega}=m D,
$$

где $D=k_{\mathrm{B}} T /(m \omega)$ - коэффициент тепловой диффузии осциллятора при $t \gg \tau$. Разумеется, здесь в роли импульса осциллятора естественно выступает импульс $p=m \omega q$ соответствующего потока броуновских осцилляторов, как это и принято в теории броуновского движения [5].

Можно ожидать, что наличие у волновой функции осциллятора в термостате фазы, зависящей от температуры во всей области ее значений $0 \leqslant T<\infty$, найдет отражение и в других характеристиках описания состояния теплового равновесия осциллятора. 


\section{6. ЗАКЛЮЧЕНИЕ}

Итак, мы показали, что состояние теплового равновесия для квантового осциллятора в термостате может быть непротиворечиво описано волновой функцией, т.е. его можно рассматривать как своеобразное чистое состояние в удвоенном гильбертовом пространстве. Вместе с тем характеристики подобного состояния, и прежде всего его фаза и связанная с ней ковариация $\sigma_{p q}$, зависят существенно от температуры, демонстрируя наличие в подобном состоянии теплового шума [16].

Наличие теплового шума обычно принято трактовать как проявление неупорядоченности в рассматриваемой системе, которая характеризуется отличной от нуля энтропией. В то же время согласно традиционным представлениям энтропия чистого состояния должна равняться нулю. На наш взгляд, это обстоятельство означает необходимость определенной корректировки стандартного формализма статистической механики, особенно при сверхнизких температурах, в частности, необходимо уточнение определения энтропии. Это позволит учесть и более тонкие особенности неупорядоченности в данном состоянии, связанные с наличием теплового шума.

Достигнутый выше результат - получение насыщенного соотношения неопределенностей Шредингера для осциллятора в термостате с помощью чистых состояний - разумеется, не снимает необходимости получить аналогичный результат с помощью смешанных состояний. Заметим в этой связи, что величина $c_{p q}$ от выбора состояния не зависит, а величина $\sigma_{p q}$ для вещественной матрицы плотности автоматически обращается в нуль. Поэтому единственная возможность устранить возникшее несоответствие в вычислениях при использовании смешанных состояний, по-видимому, связана с необходимостью модификации целостного выражения для $\mathcal{U P}$, входящего в левую часть соотношения неопределенностей Шредингера, что уже отмечалось рядом авторов [18].

Таким образом, нельзя исключить, что полученные результаты допускают и другие возможности физической интерпретации. K этому вопросу мы намерены вернуться в последующих публикациях.

Благодарности. Автор выражает благодарность участникам семинара Лаборатории теоретической физики ОИЯИ им. Н. Н. Боголюбова и рецензентам за дискуссию, а О. Н. Голубевой - за благожелательную критику и помощь в окончательном редактировании текста статьи.

\section{Список литературы}

[1] Л. Д. Ландау, Е. М. Лифшиц, Теоретическая физика, m. V. Статистическая физика. Часть 1, Физматлит, М., 2001.

[2] А. Д. Суханов, ТМФ, 125:2 (2000), 221.

[3] А. Д. Суханов, ТМФ, 132:3 (2002), 449.

[4] А. Д. Суханов, ЭЧАЯ, 32:5 (2001), 1177; J. Uffink, J. van Lith, J. Found. Phys., 28 (1998), 323.

[5] А.Д. Суханов, ТМФ, 139:1 (2004), 129.

[6] A. D. Sukhanov, "On the Global Interrelation between Quantum Dynamics and Thermodynamics", Proc. XI Int. Conf. "Problems of Quantum Field Theory" (Dubna, Russia, 
12-17 July 1998), eds. B. M. Barbashov, G. V. Efimov, A. V. Efremov, JINR, Dubna, 1999, 232.

[7] F. Bloch, Z. Phys., 74 (1932), 295.

[8] М. А. Леонтович, Введение в термодинамику. Статистическая физика, Наука, М., 1983.

[9] А. Эйнштейн, М. Смолуховский, "Несколько примеров брауновского молекулярного движения под действием внешних сил", Брауновское движение, ОНТИ, М., 1936, 205; А.И. Ансельм, Основы статистической физики и термодинамики, Наука, M., 1973.

[10] Э. Шредингер, “К принципу неопределенностей Гейзенберга", Избранные труды по квантовой механике, Наука, М., 1976, 210.

[11] В. В. Додонов, В. И. Манько, Тр. ФИАН, 183 (1987), 5.

[12] E. Nelson, Dynamical Theory of Brownian Motion, Princeton Univ. Press, Princeton, NJ, 1967.

[13] В. В. Додонов, В. И. Манько, Тр. ФИАН, 183 (1987), 71.

[14] V. V. Dodonov, J. Opt. B. Quantum Semiclass. Opt., 4 (2002), S98.

[15] Х. Умэдзава, Х. Мацумото, М. Такики, Термополевая динамика и конденсированные состояния, Мир, М., 1985.

[16] H. Umezawa, Advanced Field Theory. Micro, Macro and Thermal Physics, AIP, N.Y., 1993.

[17] Ю.Л. Климонтович, Статистическая теория открытых систем, m. 3. Физика квантовых открытых систем, Янус, М., 2001.

[18] E. P. Wigner, Z. Phys., 133 (1952), 101; E. P. Wigner, M. M. Yanase, Proc. Nat. Acad. Sci. USA, 49 (1963), 910; E. H. Lieb, Adv. Math., 11 (1973), 267; S. Luo, Z. Zhang, J. Stat. Phys., 114 (2004), 1557. 